\title{
Contribución de la gastronomía típica al desarrollo turístico del destino Cuba.
}

\section{Contribution of typical gastronomy to the tourist development of the destination Cuba.}

Julia María Espinosa Manfugás. ${ }^{1}$, Beatriz Romaní Bendig. ${ }^{2}$, Laura Hernández Navarrete. $^{3}$, \& Rocío Arias Rodríguez. ${ }^{4}$

\section{Resumen.}

La presente investigación tiene como objetivo determinar el potencial que ofrece la gastronomía típica del país y las posibles sinergias que existen entre esta y los turistas que seleccionan a Cuba como destino turístico. Se emplearon métodos teóricos y empíricos de investigación, analizando los resultados mediante el diagrama de Pareto, el test de Friedman, la prueba de Wilcoxon y el análisis discriminante, utilizando el programa estadístico SPSS versión 22. Se evidenció que el patrimonio gastronómico del destino es auténtico y variado y puede contribuir al desarrollo del producto turístico de la nación, sin embargo, en la actualidad no están creadas las condiciones para ello. Los restaurantes extrahoteleros del país no se orientan en su mayoría hacia la oferta de cocina cubana, la comercialización de los platos típicos es limitada y existe insatisfacción de los turistas con la oferta, básicamente con las variables y atributos que mayor impactan en sus expectativas.

Palabras claves: Cocina cubana, carta menú, gastronomía, restaurantes, turistas.
Abstract.
The objective of this research is to determine the potential offered by the typical gastronomy and the possible synergies that exist between it and the tourists who select Cuba as a tourist destination. Theoretical and empirical research methods were used, analyzing the results using the diagram of Pareto, the Friedman test, the Wilcoxon test and the discriminant analysis, using the statistical program SPSS, version 22. It was shown that the gastronomic heritage of the destination is authentic and varied and can

\footnotetext{
${ }^{1}$ Universidad de La Habana, Facultad de Turismo, La Habana. Cuba. julia.maria@ftur.uh.cu

${ }^{2}$ Universidad de La Habana, Facultad de Turismo, La Habana. Cuba. carolanr95@ hotmail.com

${ }^{3}$ Universidad de La Habana, Facultad de Turismo, La Habana. Cuba.

${ }^{4}$ Dirección Nacional de Gaviota. La Habana. Cuba. esp1.negocio@gaviota.cu
} 
contribute to the development of the tourism product of the nation, however at present the conditions for it are not created. The extrahotel restaurants in the country are not oriented mostly towards the offer of Cuban cuisine, the commercialization of typical dishes is limited and there is dissatisfaction of tourists with the offer, basically with the variables and attributes that have the greatest impact on their expectations.

Keywords: Cuban cuisine, menu, gastronomy, restaurants, tourists

\section{Introducción}

En los últimos años la gastronomía ha sido una vía para la creación de nuevos productos turísticos a nivel internacional, por cuanto engloba un conjunto de actividades de contenido cultural e histórico que le permiten al turista adquirir experiencias inolvidables del territorio visitado Morales, y Gómez (2006) y Oliveira (2011), proliferando la modalidad de turismo gastronómico [1; 2], la que se define por Montecinos (2016), como el flujo de "personas que durante sus viajes y estancias realizan actividades fundamentadas en el patrimonio cultural gastronómico material e inmaterial en lugares diferentes a los de su entorno habitual, por un periodo de tiempo consecutivo inferior a un año con el principal fin de consumir y disfrutar productos, servicios y experiencias gastronómicas de manera prioritaria y complementaria" [3].

A partir de lo antes expuesto y teniendo en cuenta que a pesar de tener Cuba una cocina autentica, no la explota como un atractivo turístico en toda su magnitud, el Ministerio de Turismo, organismo rector de la industria de la hospitalidad, incluyó dentro de su plan de desarrollo tareas encaminadas a valorar la situación de la cocina cubana, a fin de que en un futuro inmediato la gastronomía típica de las diferentes regiones del país, como elemento de identidad cultural, pueda constituir una modalidad del destino y así diversificar el producto turístico de la nación.

Se trata entonces de un campo abierto con amplio alcance, el cual llevó a la realización de la presente investigación, cuyo objetivo principal es determinar el potencial que ofrece la gastronomía típica del país y las posibles sinergias que existen entre esta y los turistas que seleccionan a Cuba como destino turístico, elementos que no se han abordado de manera holística en trabajos precedentes.

Los métodos de investigación empleados, unido al tratamiento estadístico de los resultados, permitieron identificar los platos distintivos de las regiones occidental, central y oriental del país (información que estaba muy dispersa) y su comercialización en los restaurantes extrahoteleros del sector no estatal y estatal pertenecientes a la Empresa Extrahotelera Palmares S.A., constituyendo este aspecto un aporte del trabajo. De igual manera se valoró la situación de la cocina cubana desde la perspectiva de los turistas que visitan el destino.

Se constató que el patrimonio gastronómico de la nación es genuino y diverso, sin embargo, los restaurantes extra-hoteleros, en su mayoría no se orientan hacia la oferta de cocina cubana, comercializándose de manera limitada los platos típicos y tradicionales del país, lo cual es apreciado por los turistas.

\section{Métodos}

Etapa 1. Identificación de los restaurantes de cocina cubana 
La identificación de los restaurantes se efectuó mediante el análisis documental. Se consultó el anuario estadístico de la ONEI (2017), y el Manual de identidad de la Empresa Extrahotelera Palmares S.A. del mismo año, con el levantamiento de restaurantes de cocina criolla, así como las páginas web enfocadas a la promoción de restaurantes (A la mesa y Cuba Paladar). Se identificaron los restaurantes teniendo en cuenta su distribución por regiones y provincias del país [4].

\section{Etapa 2. Determinación y comercialización de los platos típicos del país}

Mediante el análisis bibliográfico-documental se determinaron las ofertas típicas del destino. Se consultó la documentación relativa a la cocina cubana, expuesta por diversos autores Matamoros, (2010); Formatur, (2012) y Fernández, (2018) [5; 6; 7].

Para completar y contrastar la información obtenida a través de la revisión documental y recopilar criterios sobre la situación de la cocina cubana, se efectuó una entrevista no estructurada a 13 especialistas con más de 15 años de experiencia como profesional en la actividad de alimentos y bebidas y/o profesores relacionados con las teorías que sustentan el tema abordado. La información recopilada se estratificó por regiones (occidental, central y oriental) y provincia que integran cada una de estas, así como por grupos de alimentos: entrantes, platos principales, guarniciones y postres.

La comercialización de las ofertas identificadas se constató a partir de la información referida por Junco (2016) [8] y las páginas web enfocadas a la promoción de restaurantes (A la mesa, Cuba Paladar), así como la observación de las cartas menú de todas las instalaciones especializadas en cocina cubana de la Empresa Extrahotelera Palmares S.A. y del sector no estatal del país.

\section{Etapa 3. Mirada de los turistas hacia la comida típica cubana}

Se aplicó una encuesta a turistas extranjeros de ambos sexos, mayores de 18 años que transitaban por las principales zonas turísticas de la provincia La Habana (Centro Histórico, municipio Playa y Plaza de la Revolución). Se decidió aplicar el cuestionario en dicha provincia, por constituir el principal destino turístico de Cuba, donde se concentra aproximadamente la mitad de la demanda del país.

La cantidad de encuestados se determinó a través de un muestreo probabilístico, considerando la población infinita (mayor de 100,000), atendiendo a que la cantidad de turistas que visitaron la provincia el año anterior (2017) en los meses de enero a marzo, período en que se realizó la encuesta, fue superior a dicho valor. Se consideró un nivel de confianza del $95 \%(\alpha=0,05)$ y un error muestral del $5 \%$, obteniendo una tamaño de muestra de 384 .

El cuestionario fue elaborado en español y traducido al idioma inglés y francés, atendiendo a la nacionalidad de los principales mercados que visitan el destino, contaba de 10 preguntas, dirigidas fundamentalmente a conocer su perfil socio demográfico, motivación de viajar a Cuba, conocimiento y apreciación de la cocina cubana, evaluación a través de una escala de likert de tres categorías (satisfecho, medianamente satisfecho e insatisfecho) de las variable instalación, personal y oferta; para esta última se valoraron además los atributos (presentación de la oferta, variedad, calidad y temperatura de las 
elaboraciones y las bebidas y tamaño de la ración). Se analizaron los resultados mediante el Diagrama de Pareto.

La incidencia de las variables en las expectativas de los encuestados también fue objeto de estudio, para ello se empleó la prueba de ordenamiento reportada por Espinosa (2015), procesando los resultados a través del Test bidimensional de Friedman y en caso de existir variación estadística la prueba de comparación por rangos de Wilcoxon, para un nivel de confianza del $95 \%$. Se aplicó la prueba de reducción de dimensiones, escalamiento óptimo, para definir los atributos de la oferta que mejor discriminan [9].

Se estimó la confiabilidad del cuestionario mediante el coeficiente $\alpha$ de Cronbach, índice de consistencia que según Hernández, Fernández y Baptista, (2010), es aceptable cuando es mayor de 0,75 [10].

Se utilizó el programa estadístico SPSS para Windows (versión 22.0).

\section{Resultados.}

\section{Etapa 1. Identificación de los restaurantes de cocina cubana.}

\section{$\underline{\text { Sector no estatal }}$}

Se identificaron en el país un total de 689 restaurantes del sector no estatal, de ellos 348 ofertan platos de la cocina cubana y cubana- fusionada (cubana- internacional, cubanaitaliana, cubana- brasileña, cubana- china, entre otras); para un 50,6\%. En la figura 1 se puede observar la distribución por regiones (occidental, central y oriental), siendo la occidental la de mayor presencia con 256 instalaciones (73\%)

En el occidente es La Habana la de mayor número de instalaciones (191) para un 75\% y en la región central, Camagüey y Ciego de Ávila con 27 (39\%) y 18 (26\%) respectivamente, bastante distante de Cienfuegos con 10 (14\%), Sancti Spíritus con 8 (12\%) y Villa Clara con sólo 6 (9\%). La zona oriental es la de menor porcentaje del país y la provincia Guantánamo la que exhibe más restaurantes, 8 para un 35\%. En Santiago de Cuba y Holguín si bien la cantidad de instalaciones del sector no estatal ha crecido en los últimos años, solo se especializan en comida cubana 5 (22\%). En Las Tunas y Granma solo existen 3 y 2 restaurantes respectivamente.

\section{$\underline{\text { Sector estatal (Empresa Extrahotelera Palmares S.A.) }}$}

La Empresa Extrahotelera Palmares S.A. agrupa un total de 112 restaurantes, de ello 28 se dedican a la oferta de comida cubana y 1 a la cubana fusionada, para un total de 29 (26\%). La distribución por regiones se muestra en la figura 2. La Habana en el occidente es la provincia de mayor porcentaje (24\%), en el centro Sancti Spíritus y Ciego de Ávila y en el oriente Santiago de Cuba con un 7\%. 
Figura 1. Porcentaje de restaurantes de cocina cubana del sector no estatal

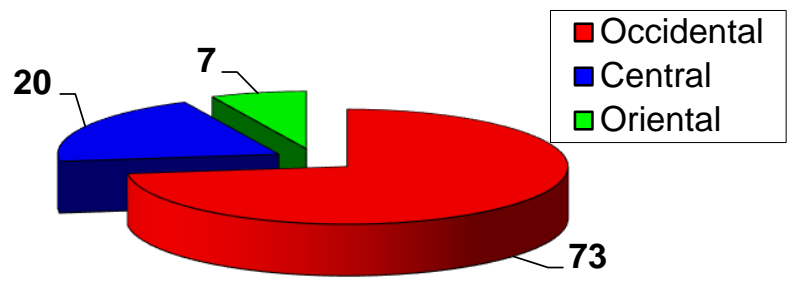

Figura 2. Porcentaje de restaurantes de cocina cubana del sector estatal

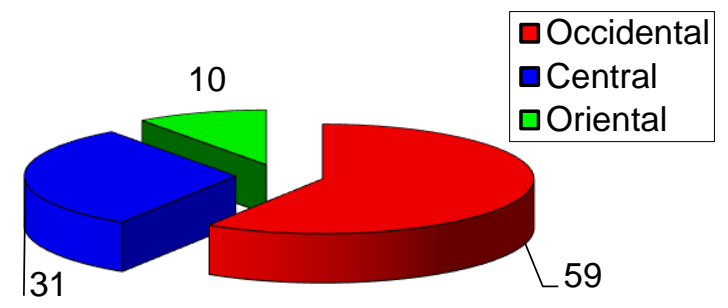

\section{Etapa 2. Determinación y comercialización de los platos típicos del país.}

Se registraron un total de 390 elaboraciones culinarias típicas del país, las cuales estratificadas por regiones y provincias se presentan en la tabla 1. La comercialización de estas se presenta en la figura 3, observándose que sólo se oferta el 39\%. Los platos principales que son los más comercializados sólo alcanzan un $48 \%$ del total.

Tabla 1. Platos típicos identificados por regiones y provincias del país.

\begin{tabular}{|c|c|c|c|c|c|c|}
\hline Región & Provincias & Entrantes & Platos principales & Guarniciones & Postres & Total \\
\hline \multirow{6}{*}{ Occidental } & Isla de la Juventud & 9 & 9 & 5 & & 23 \\
\hline & Pinar del Rio & - & 12 & - & & 12 \\
\hline & Artemisa & - & 5 & 1 & & 6 \\
\hline & Mayabeque & - & 11 & 3 & & 14 \\
\hline & La Habana & 9 & 15 & 6 & & 30 \\
\hline & Matanzas & 8 & 14 & - & & 22 \\
\hline Total & & 26 & 66 & 15 & 44 & 151 \\
\hline \multirow{4}{*}{ Central } & Cienfuegos & 8 & 14 & 5 & & 27 \\
\hline & Villa Clara & - & 3 & 1 & & 4 \\
\hline & Sancti Spíritus & 3 & 17 & - & & 20 \\
\hline & Ciego de Ávila & 10 & 24 & 1 & & 35 \\
\hline
\end{tabular}




\begin{tabular}{|c|c|c|c|c|c|c|}
\hline & Camagüey & 4 & 3 & - & & 7 \\
\hline \multirow{4}{*}{ Total } & & $\mathbf{2 5}$ & $\mathbf{6 1}$ & $\mathbf{7}$ & $\mathbf{3 2}$ & $\mathbf{1 2 5}$ \\
\hline \multirow{3}{*}{ Oriental } & Las Tunas & 2 & 19 & - & & 21 \\
\cline { 2 - 6 } & Holguín & 10 & 13 & 3 & & 26 \\
\cline { 2 - 7 } & Granma & 3 & 9 & - & & 12 \\
\cline { 2 - 7 } & $\begin{array}{c}\text { Santiago de Cuba } \\
\text { Guantánamo }\end{array}$ & 4 & 12 & - & & 16 \\
\hline Total & & $\mathbf{1 9}$ & $\mathbf{5 3}$ & $\mathbf{3}$ & $\mathbf{3 9}$ & $\mathbf{1 1 4}$ \\
\hline & & $\mathbf{7 0}$ & $\mathbf{1 8 0}$ & $\mathbf{2 5}$ & $\mathbf{1 1 5}$ & $\mathbf{3 9 0}$ \\
\hline
\end{tabular}

Figura 3. Comercialización de las ofertas típicas identificadas

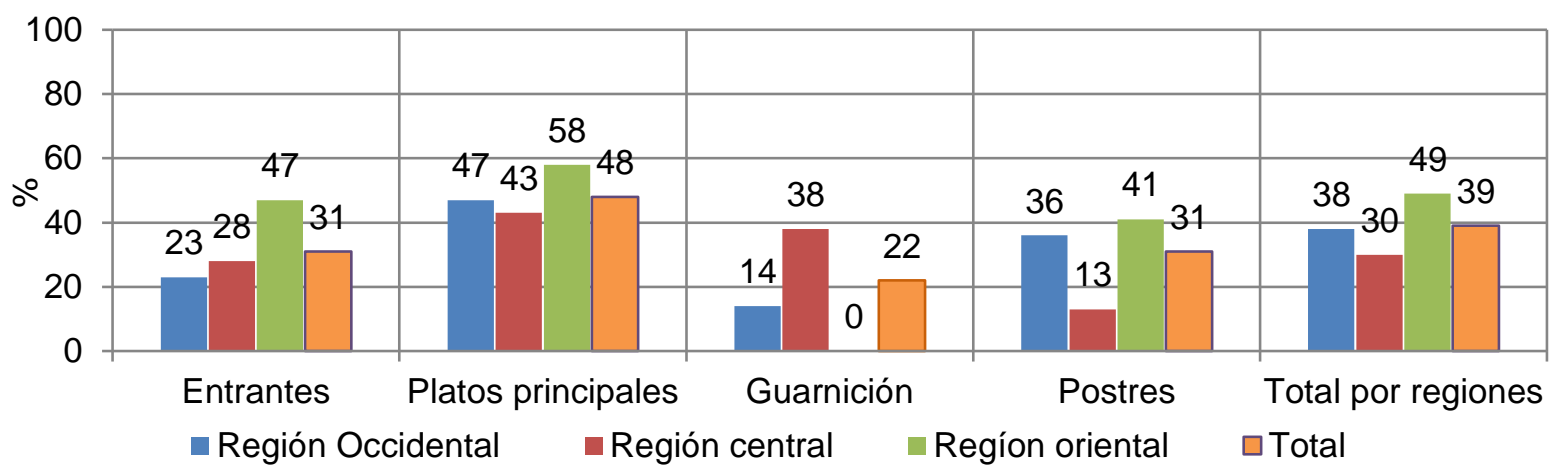

Para analizar las presentaciones de las ofertas en las cartas menú se tomó como referencia de los 379 platos típicos registrados, las 23 preparaciones reportadas por Fernández (2018) como las más tradicionales y populares [7]. Se analizaron 107 cartas menú (28 restaurantes del sector estatal y 79 del sector no estatal). Las ofertas representadas se visualizan en la Figura 4.

Figura 4. Cantidad de preparaciones tradicionales presentadas en las cartas menús de los restaurantes 


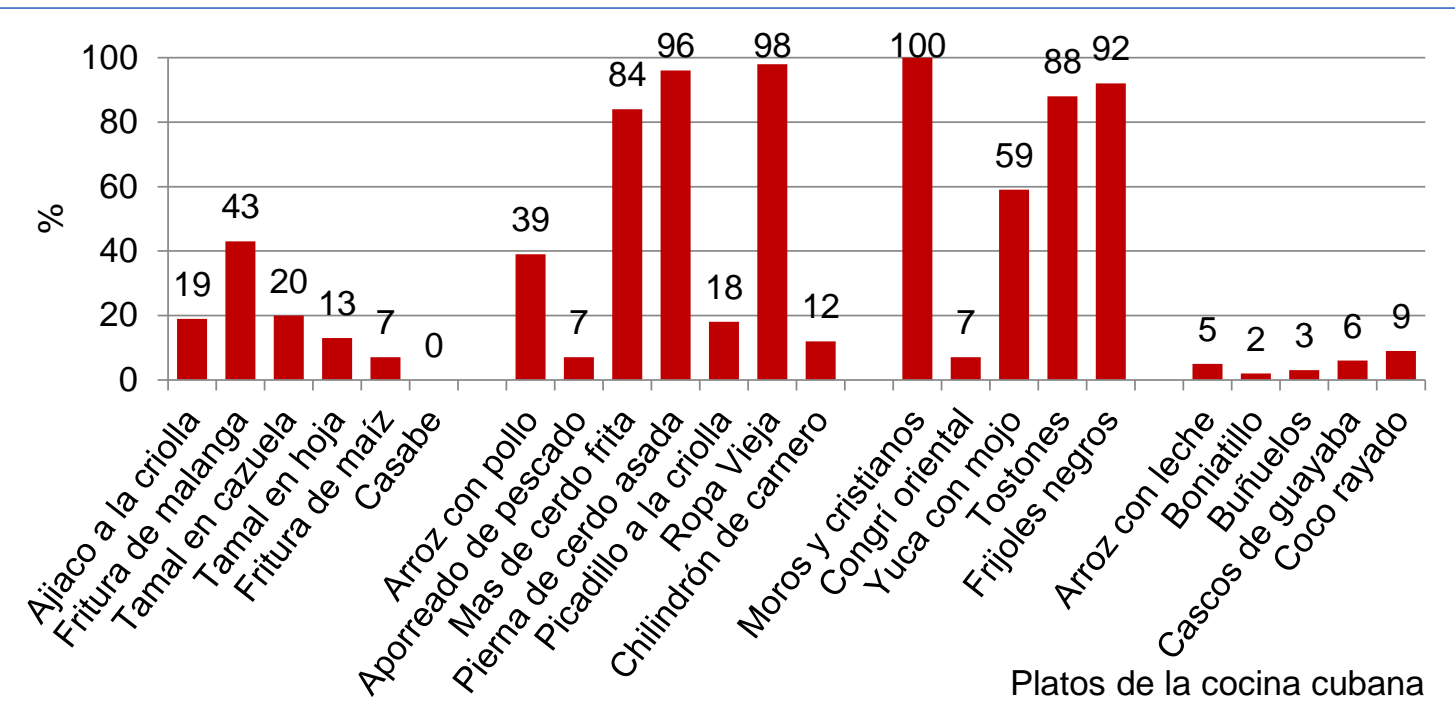

\section{Etapa 3. Mirada de los turistas hacia la comida típica cubana.}

La encuesta aplicada fue fiable, Se obtuvo un coeficiente $\alpha$ de Cronbach de 0,819, (superior a 0,75 ).

La mayoría de los turistas encuestados se encuentran en el rango de edad de 25 a 50 años para un $52,1 \%$, con un predominio ligero del sexo femenino, procedente de diversas nacionalidades, fundamentalmente estadounidenses, ingleses, españoles y canadienses. El 91,4\% alegó que era la primera vez que viajaba a Cuba. El ocio y la recreación fue el motivo de viaje fundamental de los encuestados, no así la gastronomía que sólo alcanzó un $2,3 \%$

El 37,0\% manifestó que tenía conocimiento sobre la gastronomía típica del país, no obstante, estimaron que la información que poseían era limitada. El 78,6\% durante su estancia en Cuba habían visitado al menos un restaurante de comida cubana y el $88,0 \%$ de estos manifestó haber cumplido sus expectativas.

Sobre la satisfacción de los turistas con el servicio de los restaurantes, la variable mejor evaluada fue "personal", seguida de la instalación y la oferta, el comportamiento de los atributos que componen la oferta se presenta en la figura 5.

Figura 5. Diagrama de Pareto

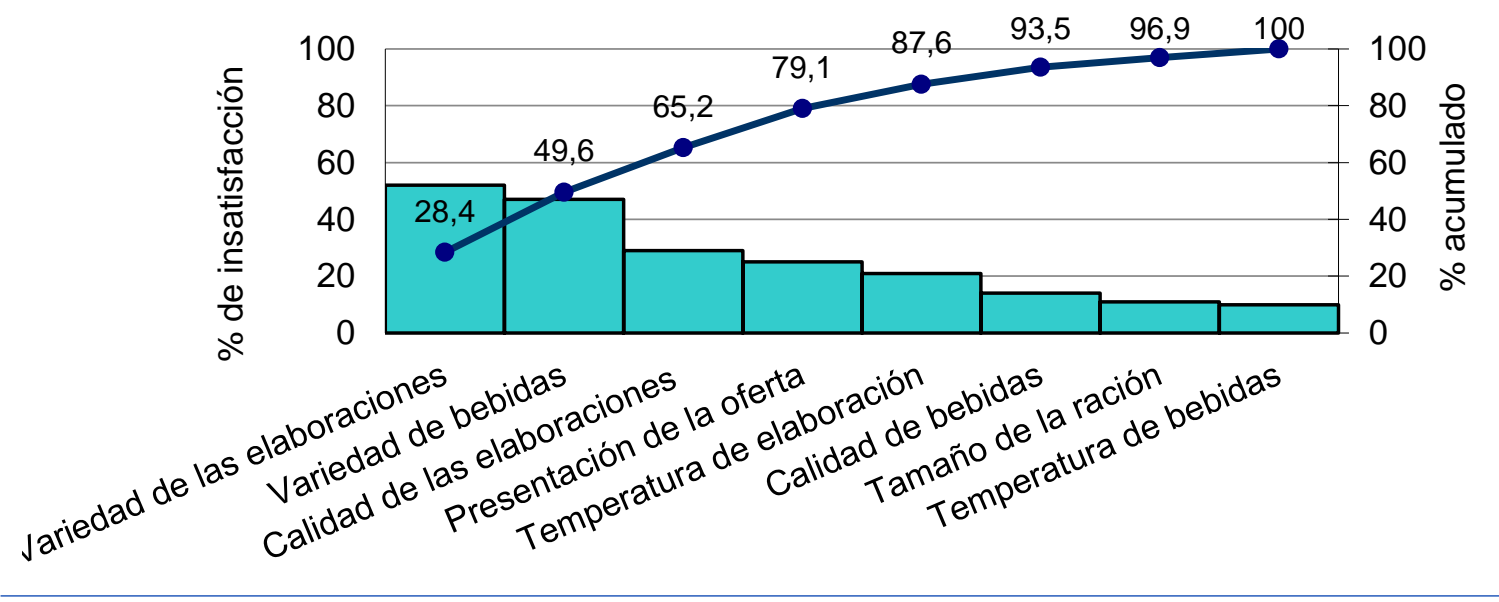


Al determinar el impacto de las variables en las expectativas de los turistas encuestados a través del Test bidimensional de Friedman, se encontró diferencia significativa, arrojando la prueba de Wilcoxon que la "oferta" es la variable más importante en relación a la "instalación" y el "personal", entre las cuales no existe variación apreciable $(p<0,05)$

La incidencia de los atributos que componen la oferta a partir de la prueba de esclamiento optimo se exhibe en la figura 6.

Figura 6. Medidas discriminantes

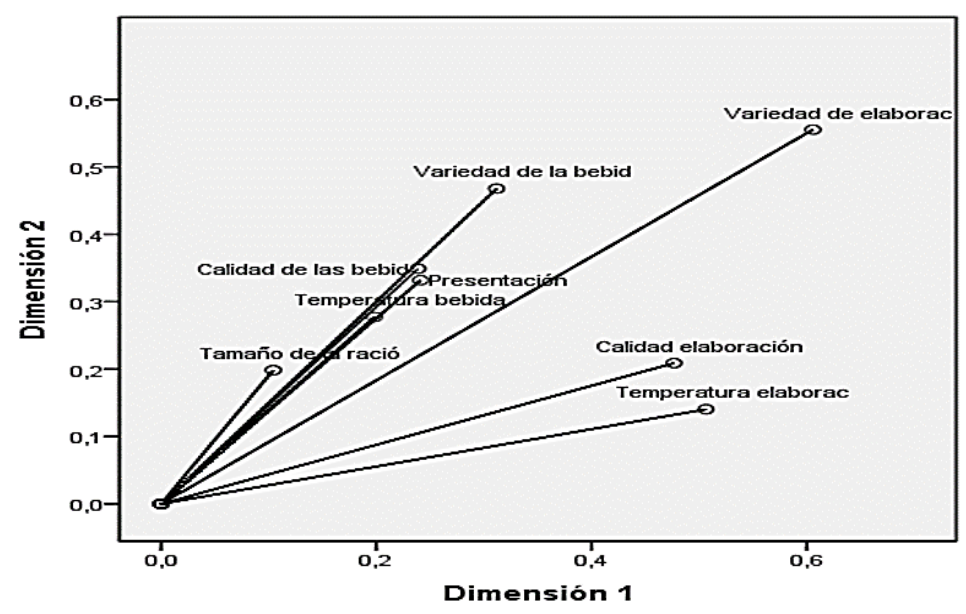

\section{Discusión}

\section{Etapa 1. Identificación de los restaurantes de cocina cubana}

Los resultados demuestran un escenario diferente en cuanto al porcentaje de restaurantes que ofertan comida cubana perteneciente al sector no estatal y la Empresa Extrahotelera Palmares S.A, evidenciándose una menor representación en el sector estatal. Situación que merece atención, por cuanto Palmares es la Empresa que representa al Ministerio de Turismo cubano. La distribución de los restaurantes por regiones es similar en ambos sectores, siendo la occidental la de mayor presencia de estos.

El análisis por provincia en cada una de las regiones demuestra un comportamiento heterogéneo, La Habana en el occidente es la provincia con más instalaciones, comportamiento esperado por ser la capital del país. De igual modo las cifras reportadas para la región central guardan relación con las características de cada una de las provincias, en cuanto a extensión territorial y población, no así en la zona oriental, la cual presentó un escenario distinto al esperado.

\section{Etapa 2. Determinación y comercialización de los platos típicos del país}


Se constató que la cocina cubana es versátil y genuina, con platos típicos representativos de las diferentes provincias del país, y de localidades específicas dentro de estas, lo que de ser explotado convenientemente permitiría diversificar la oferta y contribuiría al desarrollo turístico del destino.

La comercialización de los platos típicos en los restaurantes del país como se observa en la figura 3, es limitada, situación que según criterio de los especialistas entrevistados y la experiencia de las autoras se debe fundamentalmente a: carencia de suministros por parte de los proveedores e inestabilidad en el surtido, formación inapropiada, desmotivación y falta de creatividad del personal de cocina, unido a que en su mayoría los gestores de alimentos y bebidas no tienen conciencia de que las tradiciones culinarias regionales constituyen un elemento diferenciador y un valor agregado al negocio de la restauración.

El análisis de la cartas menú arrojó que sólo el 26\% de los platos considerados como más tradicionales y populares se presentan en más del $80 \%$ de estas, resultando el grupo de postres el de mayor dificultad. Dentro de los entrantes el "casabe", plato típico cubano heredado de los aborígenes, no forma parte de la oferta en ninguna de las cartas observadas y el "ajiaco", considerado como plato nacional, sólo se exhibe en el 19\% de estas, en cambio dentro de los platos principales la "ropa vieja" tiene un 98\% de presentación y la "masa de cerdo frita" y "pierna de cerdo asada" 84 \% y $89 \%$ respectivamente, estas últimas referidas por la población de manera indistinta y errónea como el plato nacional. En el caso de las guarniciones: los moros y cristianos, frijoles negros y tostones presentan una situación favorable, no así el congrí que apenas se oferta.

\section{Etapa 3. Mirada de los turistas hacia la comida típica cubana}

Si bien los encuestados no tenían conocimiento de la gastronomía típica del país, la mayoría visitó restaurantes de comida cubana, aludiendo que cuando viajan les gusta disfrutar de la comida típica del lugar que visitan, planteamiento que confirma lo expuesto por Clemente y col., (2008) [11].

Como se aprecia en el Diagrama de Pareto de los atributos que componen la oferta, 4 se consideran vitales, obteniéndose las mayores insatisfacciones para la variedad de la oferta, lo que puede atribuirse a las causas antes expuestas, con prevalencia de la situación de los proveedores.

Es preocupante el hecho de que sea la oferta la variable de más dificultad y dentro de esta la variedad, por ser justamente esta variable y atributo respectivamente los que más discriminan e impactan en la expectativa de los turistas encuestados, resultado que corrobora lo reportado por Rochat (2001), quien expuso que el menú es probablemente el factor más significativo para los clientes que reciben servicio de alimentos y bebidos por ser el órgano de comunicación entre estos y el restaurante [12].

\section{Conclusiones}

1. Existen potencialidades en el destino para que la gastronomía sea considerada un atractivo turístico y contribuya a diversificar el producto turístico de la nación., pero aún no están creadas las condiciones para ello. 
2. Los restaurantes extrahoteleros del destino no se orientan en su mayoría hacia la oferta de cocina cubana, siendo el sector no estatal el más representado, con una proyección favorable en pos de mantener viva las tradiciones culinarias del país.

3. El patrimonio gastronómico del destino es auténtico y representa los valores tradicionales de la nación, sin embargo la comercialización de los platos típicos que lo integran es limitada. Las ofertas consideradas como más tradicionales y populares se expenden en un número reducido de restaurantes.

4. Si bien la gastronomía no ocupa un lugar preponderante en la decisión de los viajeros al seleccionar el destino Cuba, existe un vínculo entre esta y los turistas, quienes manifestaron que dentro de sus expectativas se encontraba degustar la comida típica del país.

5. La mayor insatisfacción de los turistas encuestados con el servicio de los restaurantes de comida cubana estuvo en correspondencia con las dimensiones de mayor impacto en sus expectativas. La variable "oferta" fue la más devaluada y dentro de esta los atributos variedad y calidad de las elaboraciones.

\section{Referencias Bibliográficas}

MORALES, R. y GÓMEZ, V., Gastronomía típica margariteña como atractivo turístico del municipio Antolín del Campo. Estado Nueva Esparta. Revista de turismo y patrimonio cultural. PASOS. 4(2)255-269. (2006). ISSN 1695-7121

OLIVEIRA, Simao., La gastronomía como atractivo turístico primario de un destino. El Turismo Gastronómico en Mealhada-Portugal. Revista Estudios y Perspectivas en Turismo. 20(1)738-752. (2011).ISSN 1851-1732

MONTECINOS Antonio Turismo Gastronómico Sostenible: Planificación de Servicios, Restaurantes, Rutas, Productos Y Destinos”, “Turismo Gastronómico Sostenible: Planificación de Servicios, Restaurantes, Rutas, Productos Y Destinos”. México. Editorial CEGAHO. 2016.

ONEI (2017), Anuario Estadístico de Cuba. Edición 2017. Disponible en: http://www.one.cu. Fecha de consulta: (03-01-2018).

MATAMOROS TRABA A. La cocina cubana. Origen y evolución. EAEHT. Ediciones Balcón. La Habana. Cuba. 2010

FORMATUR La cocina tradicional cubana. La Habana. Cuba Ediciones Balcón 2012.

FERNÁNDEZ MONTE, E. Los platos emblemáticos de la cocina cubana. La Habana Cuba. Editorial Arte Chef. 2018.

JUNCO, J. Proceso de formación de la cocina cubana. Influencias foráneas. Federación de Asociaciones culinarias de la República de Cuba. La Habana, Cuba. Editorial Arte chef .2016. 
ESPINOSA, MANFUGAS, J.M. Evaluación Sensorial. La Habana. Cuba, Editorial Félix Varela. 2015. ISBN 978-959-07-1956-1

HERNÁNDEZ, R.; FERNÁNDEZ, C. y BAPTISTA, M. P. Metodología de la investigación (quinta edición) México. Editorial McGraw Hill Interamericana de México, S.A. 2010. ISBN: 978-607-15-0291-9

CLEMENTE, José; ROIG, B.; VALENCIA, Sara; RABADAN; María y MARTINEZ, Cristina. Actitud hacia la gastronomía local de los turistas: dimensiones y segmentación de mercados. Revista de turismo y patrimonio cultural. PASOS. 6(2):189-198. 2008. ISSN 1695-7121.

ROCHAT, M. Marketing y gestión de la restauración. Barcelona. España. Editorial Gestión 2000, S.A. 2001. 


\section{Para citar el artículo indexado.}

Espinosa J., Romaní B., Hernández L. \& Arias R. (2017). Contribución de la gastronomía típica al desarrollo turístico del destino Cuba. Revista electrónica Explorador Digital 1(1), 28-38. Recuperado desde:

http://cienciadigital.org/revistacienciadigital2/index.php/exploradordigital/article/view/313/7 $\underline{25}$

\section{Ciencia \\ Digital \\ Editorial}

El artículo que se publica es de exclusiva responsabilidad de los autores y no necesariamente reflejan el pensamiento de la Revista Explorador Digital.

El articulo queda en propiedad de la revista y, por tanto, su publicación parcial y/o total en otro medio tiene que ser autorizado por el director o editor de la Revista Explorador Digital.
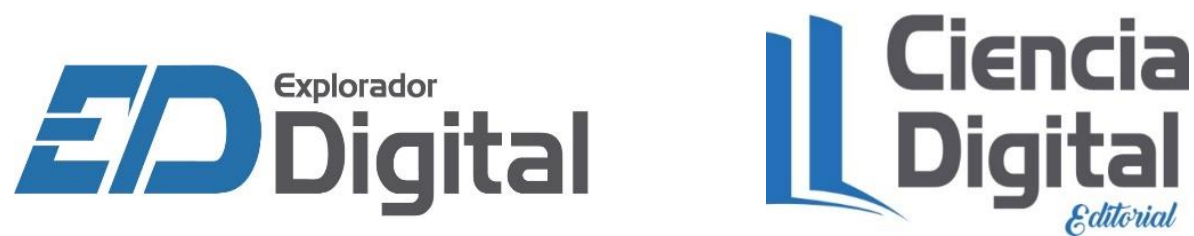